\title{
Estrés académico en estudiantes universitarios ${ }^{*}$
}

\author{
Academic stress in university students
}

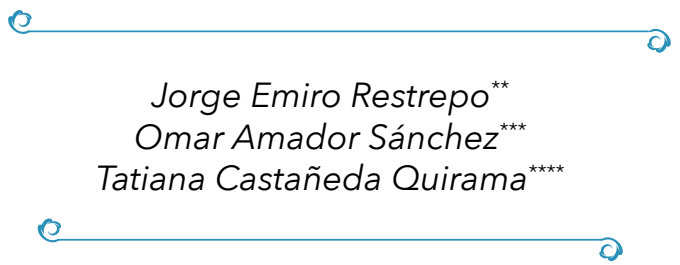

Recibido 25. 03. 2020 • Arbitrado 18. 04. 2020 •

Aprobado 15.05. 2020

Este artículo es un producto derivado del proyecto de investigación "Relación entre la ideación suicida con depresión, estrés académico y consumo de sustancias en estudiantes universitarios del área metropolitana de $\mathrm{Me}$ dellín" realizado entre la Universidad Católica Luis Amigó, el Tecnológico de Antioquia y la Corporación Universitaria Minuto de Dios-Bello.

* Doctor en neuropsicología. Profesor Asociado, Facultad de Educación y Ciencias Sociales. Tecnológico de Antioquia, Medellín, Colombia, jorge. restrepo67@tdea.edu.co_ORCID: http:/ / orcid.org/0000-0001-8790-7454

*** Magíster en psicología. Profesor investigador. Facultad de Psicología y Ciencias Sociales. Universidad Católica Luis Amigó, Medellín, Colombia, omar.amadorsa@amigo.edu.co ORCID: http:/ / orcid.org/0000-00016013-8480

**** Magíster en psicología. Profesora investigadora. Facultad de Sociedad, Cultura y Creatividad. Politécnico Grancolombiano, Medellín, Colombia, lcastaneda@ poligran.edu.co ORCID: http://orcid. org/0000-0002-1132-4221

\section{Resumen}

Objetivo: analizar el estrés académico en estudiantes universitarios. Para este propósito se definieron tres objetivos específicos: 1) identificar cuáles situaciones o condiciones del ambiente académico resultan más estresantes, 2) caracterizar las reacciones fisiológicas, psicológicas y comportamentales, y 3) conocer las estrategias de afrontamiento más frecuentes.

Método: se desarrolló una investigación con enfoque cuantitativo, transversal y de nivel descriptivo con una muestra de 450 estudiantes de tres universidades de Medellín y el Valle de Aburrá (dos instituciones privadas y una pública) utilizando el Inventario SISCO del Estrés Académico.

Resultados: para la categoría Situaciones y condiciones estresantes, el ítem con más porcentaje en la clasificación alto fue el de Sobrecarga de tareas y trabajos escolares. Para la categoría Reacciones físicas, el ítem con más porcentaje en la clasificación alto fue el de Inquietud (este ítem presentó diferencias según la edad). Para la categoría Reacciones psicológicas, el ítem con más 
porcentaje en la clasificación alto fue el de Somnolencia o mayor necesidad de dormir. Para la categoría Reacciones comportamentales, el ítem con más porcentaje en la clasificación alto fue el de Aumento o reducción del consumo de alimentos (este ítem presentó diferencias según el semestre). Para la categoría Estrategias de afrontamiento, el ítem con más porcentaje en la clasificación alto fue el de Habilidad asertiva.

Discusión: este conjunto de reacciones no hará más que menguar y perjudicar la capacidad intelectual y el desempeño académico de los estudiantes y, en muchos casos, el estrés académico (en conjunción con otras problemáticas) podría derivar en fracaso y deserción. Incluso podrían entretejer una trampa muy riesgosa para la salud y el proyecto de vida de personas jóvenes, en su mayoría menores de 25 años.

Palabras clave: estrés académico, estudiantes universitarios, salud mental.

\section{Abstract}

Objective: to analyze academic stress in university students. For this purpose, three specific objectives were defined: 1 ) identify specific situations or conditions of the most stressful academic environment, 2) characterize the physiological, psychological and behavioral reactions, and 3) know the most frequent coping strategies.

Method: an investigation with a quantitative, transversal and descriptive level approach was developed with a sample of 450 students from three universities in Medellín and the Aburrá Valley (two private institutions and one public) using the SISCO Inventory of Academic Stress.

Results: for the Stressful situations and conditions category, the item with the highest percentage in the high ranking was the Overload of homework and schoolwork. For the Physical reactions category, the item with the highest percentage in the high ranking was Restlessness (this item differentiated according to age). For the Psychological reactions category, the item with the highest percentage in the high ranking was that of Somnolence or the greatest need for sleep. For the Behavioral reactions category, the item with the highest percentage in the high ranking was the increase or reduction of food consumption (this item differs according to the semester). For the Coping Strategies category, the item with the highest percentage in the high ranking was Assertive Skill.

Discussion: from an educational perspective, this interest is justified since that set of reactions will only diminish and impair the intellectual capacity and academic performance of students and, in many cases, academic stress (in conjunction with other problems) It could lead to failure and desertion. It could also be a very risky trap for the health and life project of young people, mostly under 25.

Keywords: academic stress, university students, mental health. 


\section{Introducción}

La transición a la vida universitaria requiere que los estudiantes realicen esfuerzos por adaptarse a un nuevo rol, normas, responsabilidades y demandas académicas, en un ambiente que tiene un trasfondo de mayor competitividad. El estudiante universitario se enfrenta a situaciones estresantes relacionadas con los horarios, los exámenes, la espera de calificaciones o la incertidumbre sobre su futuro como profesional. Además, según sean sus expectativas académicas y condiciones psicosociales (problemas financieros, familiares, tiempo limitado para compartir con familia pareja o amigos), habrá mayor probabilidad de que experimente mayores niveles de estrés (Peñacoba y Moreno-Jiménez, 1999).

El estrés es una reacción comportamental, psicológica y/o fisiológica que se presenta como respuesta a un estímulo, situación o contexto que es valorado como riesgoso o demandante (Lazarus \& Folkman, 1986). En el contexto universitario hay estímulos y situaciones que podrían resultar estresantes para los estudiantes (Reddy, Menon \& Thattil, 2018). Al estrés que se presenta como respuesta a los estímulos o situaciones académicas, y que ocasionan o requieren, en algunos casos, la implementación de estrategias para afrontar la reacción, se les conoce como estrés académico (Barraza, 2003). Los estudios en esta materia han permitido establecer, por ejemplo, que la sobrecarga académica, la falta de tiempo para cumplir con las actividades y la realización de un examen son algunas de las situaciones que con mayor frecuencia generan estrés en los estudiantes universitarios (Berrío y Mazo, 2011).

A diferencia de los resultados investigativos de otros campos de estudio del estrés, existen pocas investigaciones en Colombia que aborden de forma específica y rigurosa el impacto que tiene el sistema de educación sobre los estudiantes universitarios, pese a que las demandas académicas y las actividades implicadas en este contexto efectivamente tienen repercusiones negativas sobre la salud, el bienestar y el rendimiento del estudiante (Muñoz, 2004). Teniendo en cuenta entonces que el ingreso a la vida universitaria podría convertirse en un factor importante de estrés, es de relevancia considerar cuáles son las principales causas y efectos de esta emoción, ya que estas repercuten directamente en el bienestar del estudiante y, por ende, en el éxito durante su formación profesional, por lo cual es de suma importancia implementar estrategias a nivel institucional que permitan prevenir o aminorar sus efectos (Jerez y Oyarzo, 2015).

Se han realizado varias investigaciones sobre este tema en educación secundaria. Otras se han realizado con población universitaria de diferentes 
programas académicos, como psicología, medicina, enfermería, o universitarios en general. Estos estudios se han desarrollado mayoritariamente en México y España. En Colombia, con población universitaria, pueden mencionarse las investigaciones de Parra-Osorio, Rojas-Castaño, Patiño-Córdoba, CaicedoAusecha y Valderrama-Aguirre (2015) sobre estrés académico y burnout en Cali; la de Mazo, Londoño y Gutiérrez (2013) sobre los niveles de estrés académico en Medellín; las de Gutiérrez et al. (2010) y Montoya et al. (2010) sobre depresión y estrés académico en Medellín; y la de Bermúdez et al. (2006) sobre el rendimiento académico y el estrés académico en Bucaramanga.

Los efectos del estrés pueden ir desde una incomodidad imperceptible con mínimas o inexistentes consecuencias hasta alteraciones de la salud física y psicológica con repercusiones sobre la vida personal, social y laboral (Feldman et al., 2008; Martín, 2007). En el ámbito académico, el estrés académico se ha asociado con la depresión (Gutiérrez et al., 2010), la depresión y la ideación suicida (Restrepo et al., 2018), la ansiedad (Castillo, Chacón de la Cruz y Díaz-Véliz, 2016; Pulido et al., 2011) y el estrés crónico (PozosRadillo, Preciado-Serrano, Acosta-Fernández, Aguilera-Velasco \& DelgadoGarcía, 2014). Sin desestimar los efectos del estrés sobre la salud, uno de los aspectos más relevantes está asociado con la posibilidad de que se produzcan consecuencias sobre el rendimiento académico que puedan conllevar al fracaso y la deserción escolar (Fernández-Hileman, Corengia y Durand, 2014).

Ahora bien, con relación a los estresores académicos, estos podrían definirse como los factores del ambiente educativo que generan presión o sobrecarga al estudiante. Sobre este aspecto, Awé, Gaither, Crawford \& Tieman (2016) indican que los niveles elevados de estrés estudiantil repercuten negativamente en el rendimiento escolar, además, señalan que estresores como: la carga académica, las largas horas de estudio, los exámenes, las calificaciones, el escaso tiempo libre y la dificultad que representa para el estudiante el trabajo de clase, hacen parte de los factores causales de este tipo de estrés. A lo anterior, Rosales (2016) agrega que los hábitos de estudio son otra de las variables asociadas a este fenómeno.

Aunque los hallazgos del efecto del estrés académico sobre el rendimiento académico no son concluyentes (Elias, Ping \& Abdullah, 2011; Caldera, Pulido y Martínez, 2007; Domínguez, Guerrero y Domínguez, 2015), sí hay suficiente evidencia desde la neurociencia cognitiva para respaldar la tesis de que el estrés tiene una influencia negativa sobre la atención, la memoria y, en general, sobre el aprendizaje (Vogel \& Schwabe, 2016). Ciertamente, esta influencia estará atemperada por variables individuales, familiares y socioeconómicas. Sin embargo, sea que interfiera mucho o poco con el desempeño 
académico, es una variable que debe ser considerada debido a los potenciales impactos que puede generar sobre el proyecto académico de los estudiantes y sobre su calidad de vida en general.

El estrés académico podría definirse, entonces, como: "un estado producido cuando el estudiante percibe negativamente (distrés) las demandas de su entorno" (Zárate, Soto, Castro y Quintero, 2017, p. 92). Es decir, el estudiante considera como angustiantes la mayoría de las situaciones a las que se enfrenta en su proceso formativo, ya que atribuye que sus estrategias de afrontamiento son insuficientes o poco efectivas para hacerle frente a las exigencias de su medio escolar, lo que favorece, a su vez, la aparición de reacciones como agotamiento físico, ansiedad, insomnio, bajo rendimiento académico, desinterés por la carrera, ausentismo e incluso deserción escolar. Como bien lo señala Casuso (2011), la respuesta al estrés en el ámbito universitario depende de la disposición del estudiante para hacer frente a las demandas que impone el medio educativo. Es importante resaltar que las estrategias de afrontamiento pueden ser consideradas como efectos y variables que modulan los niveles de estrés en el ámbito universitario.

$\mathrm{Al}$ respecto, Casuso (2011), señaló que, dentro de las variables moduladoras del estrés académico, la percepción de autoeficacia de los estudiantes son factores claves tanto en la aparición de respuestas de estrés, como en el proceso valorativo y de afrontamiento del mismo. Es posible que aquellos alumnos con mayores niveles de autoeficacia, en comparación con los que se perciben como menos eficaces, generen expectativas más elevadas sobre su desempeño, ya que tienen mayor confianza en sus capacidades y valoran el entorno académico como menos amenazante. Esto les permite que afronten las situaciones con más probabilidad de adaptarse con éxito a las demandas académicas y que experimenten mejores niveles de bienestar general. A lo anterior, cabe agregar que los compromisos o metas de los estudiantes, entendidos como las razones o motivos para ingresar a la universidad, también parecen mediar en la percepción del estrés, por lo cual, la orientación al logro es una de las variables determinantes como factor protector o de riesgo frente al estrés escolar.

El estrés académico está relacionado con experiencias que exige la universidad y que son indispensables para el logro de los retos planteados desde el punto de vista social, económico e institucional. En palabras de Zárate et al. (2017), el estudiante considera angustiante muchas de las situaciones a las que se enfrenta durante la formación como profesional. En la mayoría de las ocasiones sus estrategias de afrontamiento son insuficientes o poco efectivas, lo que contribuye, a su vez, a la aparición de inquietud, agotamiento físico, insomnio, bajo rendimiento académico, motivación disminuida, ausentismo 
y deserción escolar. Sumado a esto, Suárez-Montes y Díaz-Subieta (2014) apoyan la tesis de que la vulnerabilidad al estrés está influenciada por el temperamento, las habilidades de afrontamiento y la red de apoyo social, que se hacen también evidentes tanto en las manifestaciones físicas, como conductuales de cada individuo, entre las que se encuentran el aislamiento, el desgano, ademanes nerviosos, inquietud. En cuanto a las respuestas psicológicas destacan la incapacidad para concentrarse, la irritabilidad y la pérdida de confianza en sí mismo, junto con la incapacidad de tomar decisiones.

Los pocos estudios que han analizado el estrés académico en población universitaria colombiana se han concentrado en conocer el comportamiento de esta variable en relación con alguna otra, como la depresión, la ansiedad o el rendimiento académico. En el presente estudio se pretendió ampliar y profundizar la investigación de Mazo et al. (2013) que realizaron con estudiantes de una universidad privada de Medellín. No es técnicamente un análisis comparativo, ya que ese estudio utilizó un instrumento de medición diferente. Lo que se pretendió, fue realizar un estudio descriptivo que incluyera estudiantes de universidad pública y privada, y profundizar en el tema de las estrategias de afrontamiento, que no había sido abordado hasta ahora.

\section{Metodología}

\section{Diseño}

El objetivo general del proyecto fue analizar el estrés académico en estudiantes universitarios. Para este propósito se definieron tres objetivos específicos: 1) identificar cuáles situaciones o condiciones del ambiente académico resultan más estresantes, 2) caracterizar las reacciones fisiológicas, psicológicas y comportamentales, y 3) conocer las estrategias de afrontamiento más frecuentes. Se desarrolló una investigación con enfoque cuantitativo, transversal y de nivel descriptivo con una muestra de 450 estudiantes de tres universidades de Medellín y el Valle de Aburrá (dos instituciones privadas y una pública). El único criterio de exclusión fue el de tener un diagnóstico psicopatológico realizado por psicólogo clínico o psiquiatra. Los criterios de inclusión fueron: ser mayor de edad y estar matriculado en cualquier programa ofrecido por la institución.

\section{Instrumento}

Inventario SISCO del Estrés Académico (Barraza, 2007, 2008): diseñado para medir la frecuencia en la que una serie de situaciones académicas generan estrés 
y la frecuencia en la que se presentan cierto tipo de reacciones psicológicas, fisiológicas y comportamentales ante esas situaciones. Adicionalmente, valora el tipo de estrategias de afrontamiento utilizadas para responder al estrés. Es un autoinforme constituido por 37 ítems con respuesta tipo Likert agrupados en tres categorías: situaciones estresantes, reacciones y estrategias. Barraza (2007a) reportó un alfa de 0.9 para la confiabilidad global y un 0.87 para la confiabilidad por mitades. En Colombia, fue validado por Malo, Guerty, Cáceres y Peña (2010) en adultos jóvenes estudiantes universitarios quienes mostraron un alfa adecuado para la confiabilidad y valores apropiados para la validez (análisis factorial y grupos contrastados).

\section{Procedimiento}

Se realizó un muestreo no probabilístico por conveniencia. Todos los estudiantes recibieron la información sobre el estudio y firmaron el consentimiento informado. El proyecto fue revisado y aprobado por el Comité de Bioética de la Universidad Católica Luis Amigó -Medellín. La investigación se realizó cumpliendo con lo establecido en la Ley 1090 de 2006 del Colegio Colombiano de Psicólogos que reglamenta el ejercicio de la profesión de la psicología y establece el correspondiente código deontológico y bioético, así como la Resolución 8430 de 1993 del Ministerio de Salud por la cual se establecen las normas científicas, técnicas y administrativas para la investigación en salud. El instrumento fue aplicado en las aulas de clase por auxiliares de investigación que fueron capacitados por los investigadores. Los resultados fueron analizados mediante el software SPSS. v. 24. Se utilizó estadística descriptiva y pruebas de independencia (chi-cuadrado) para analizar los datos.

\section{Resultados}

El 52,2\% de los participantes estaba matriculado en el programa de Psicología. El otro $48 \%$ de los participantes estaba matriculado en alguno de los otros veintisiete programas indicados en la Tabla 1. Además del programa de Psicología, la mayor frecuencia de los participantes se presentó los pregrados de Comunicación Social-Periodismo, Trabajo Social, Ingeniería Ambiental, Contaduría Pública, Comercio Exterior, Licenciatura en Preescolar y Comunicación Social. Estos siete programas reunieron el 30,4\% de los participantes. El 17,4\% restante estuvo distribuido entre los otros veinte programas, algunos de los cuales solo tuvieron un participante. 
Tabla 1. Frecuencias por programa académico

\begin{tabular}{|c|c|}
\hline Programa & Frecuencia \\
\hline Psicología & 222 \\
\hline Comunicación Social-Periodismo & 45 \\
\hline Trabajo Social & 29 \\
\hline Ingeniería Ambiental & 19 \\
\hline Contaduría Pública & 18 \\
\hline Comercio Exterior & 13 \\
\hline Licenciatura en Preescolar & 11 \\
\hline Comunicación Social & 10 \\
\hline Criminalística & 9 \\
\hline Admón. de Empresas & 8 \\
\hline Licenciatura en Inglés & 8 \\
\hline No registra & 8 \\
\hline Gastronomía & 6 \\
\hline Admón. Financiera & 5 \\
\hline Derecho & 5 \\
\hline Investigación Judicial & 5 \\
\hline Negocios Internacionales & 5 \\
\hline Arquitectura & 4 \\
\hline Ingeniería de Sistemas & 4 \\
\hline Lengua Castellana & 4 \\
\hline Gestión Financiera & 3 \\
\hline Tanatopraxia & 2 \\
\hline Actividad Física y Deportes & 1 \\
\hline Diseño Gráfico & 1 \\
\hline Histocitotecnología & 1 \\
\hline Ingeniería de Software & 1 \\
\hline Tecnología Agroambiental & 1 \\
\hline Tecnología en Sistemas & 1 \\
\hline Teología & 1 \\
\hline Total & 450 \\
\hline
\end{tabular}

En la Tabla 2 se presentan los estadísticos descriptivos de la muestra. La composición fue relativamente homogénea en cuanto al tipo de institución, pero hubo más mujeres que hombres. El rango de edad de los participantes fue de 18 a 24 años. El 79,4\% de la muestra tenía entre 18 y 21 . El 97\% de los estudiantes pertenecen a los estratos $1,2,3$ y 4, con el porcentaje más alto de la muestra entre los estratos 3 y 4 . Se incluyeron estudiantes de todos los semestres, pero el porcentaje más alto estuvo entre el cuarto y el sexto. El 
85,4\% de los participantes tenía la condición de dedicación exclusiva al estudio, es decir, estudiante que no trabaja.

Tabla 2. Estadísticos descriptivos de la muestra

\begin{tabular}{lc}
\hline & Porcentaje \\
\hline Institución & \\
Pública & $51,7 \%$ \\
Privada & $49,3 \%$ \\
Sexo & \\
Hombre & $26,3 \%$ \\
Mujer & $73,7 \%$ \\
Edad & \\
$18-19$ & $42,3 \%$ \\
$20-21$ & $37,1 \%$ \\
$22-24$ & $20,6 \%$ \\
Estrato Socioeconómico & \\
$1-2$ & $47,2 \%$ \\
$3-4$ & $49,9 \%$ \\
$5-6$ & $3,0 \%$ \\
Semestre & \\
$1-3$ & $33,3 \%$ \\
$4-6$ & $53,9 \%$ \\
$7-10$ & $12,7 \%$ \\
Ocupación & \\
Solo estudiante & $85,4 \%$ \\
Empleado & $14,6 \%$ \\
\hline
\end{tabular}

En la Tabla 3 están los resultados del Inventario SISCO del Estrés Académico para cada uno de los ítems expresados en porcentajes de acuerdo con tres niveles (alto, medio, bajo). Estos niveles se crearon para agrupar las opciones de respuesta originales del instrumento, que estaban en una clasificación Likert de cinco opciones: nunca, rara vez, algunas veces, casi siempre y siempre. Las dos primeras opciones se clasificaron como bajo, la intermedia se clasificó como medio y las dos últimas, como alto. Para la categoría Situaciones y condiciones estresantes, el ítem con más porcentaje en la clasificación alto fue el de Sobrecarga de tareas y trabajos escolares y el ítem con más porcentaje en bajo fue el de La competencia con los compañeros del grupo (este ítem presentó diferencias según la ocupación $\left(\chi^{2}=12,35 p=0,015\right)$ para $\left.p \leq 0,05\right)$. 
Para la categoría Reacciones físicas, el ítem con más porcentaje en la clasificación alto fue el de Inquietud (este ítem presentó diferencias según la edad $\left(\chi^{2}=9,98 p=0,041\right)$ y el ítem con más porcentaje en bajo fue el de Sentimientos de agresividad o aumento de irritabilidad. Para la categoría Reacciones psicológicas, el ítem con más porcentaje en la clasificación alto fue el de Somnolencia o mayor necesidad de dormir y el ítem con más porcentaje en bajo fue el de Problemas de digestión, dolor abdominal o diarrea (este ítem presentó diferencias según la ocupación $\left(\chi^{2}=12,54 p=0,014\right)$ para $\left.p \leq 0,05\right)$. Para la categoría Reacciones comportamentales, el ítem con más porcentaje en la clasificación alto fue el de Aumento o reducción del consumo de alimentos (este ítem presentó diferencias según el semestre $\left(\chi^{2}=11,63 p=0,020\right)$ para $p \leq 0,05)$ y el ítem con más porcentaje en bajo fue el de Conflictos o tendencia a polemizar o discutir.

Para la categoría Estrategias de afrontamiento, el ítem con más porcentaje en la clasificación "alto" fue el de Habilidad asertiva y el ítem con más porcentaje en bajo fue el de Uso de psicofármacos. Las pruebas de chi-cuadrado $(p \leq 0,05)$, para establecer dependencia de los ítems en función del tipo de institución, sexo, edad, estrato y ocupación, arrojaron los siguientes resultados, además de los ya mencionados: para el ítem Trastornos del sueño, diferencias según el semestre $\left(\chi^{2}=9,76 p=0,045\right)$, para Fatiga crónica, diferencias según el estrato socioeconómico $\left(\chi^{2}=10,14 p=0,038\right)$, para Elogios a sí mismo, diferencias según la edad $\left(\chi^{2}=10,42 p=0,034\right)$, para La religiosidad, diferencias según el estrato socioeconómico $\left(\chi^{2}=10,13 p=0,038\right)$, y para Búsqueda de información sobre la situación, diferencias según la institución $\left(\chi^{2}=12,1\right.$ $p=0,007)$. No hubo diferencias estadísticamente significativas en ningún ítem según el sexo.

Tabla 3. Porcentajes clasificados para cada item

\begin{tabular}{lccc}
\hline & Baja & Media & Alta \\
\hline Situaciones y condiciones estresantes & & & \\
La competencia con los compañeros del grupo $^{1}$ & $65,7 \%$ & $28,4 \%$ & $5,9 \%$ \\
Sobrecarga de tareas y trabajos escolares & $17,0 \%$ & $47,3 \%$ & $35,7 \%$ \\
Las evaluaciones de los profesores & $24,3 \%$ & $46,8 \%$ & $28,9 \%$ \\
El tipo de trabajos que te piden los profesores & $28,4 \%$ & $41,1 \%$ & $30,5 \%$ \\
No entender los temas que se abordan en la clase & $46,8 \%$ & $34,9 \%$ & $18,4 \%$ \\
Participación en clases & $39,2 \%$ & $33,0 \%$ & $27,8 \%$ \\
Tiempo limitado para hacer el trabajo & $25,1 \%$ & $43,5 \%$ & $31,4 \%$
\end{tabular}




\begin{tabular}{|c|c|c|c|}
\hline & Baja & Media & Alta \\
\hline \multicolumn{4}{|l|}{ Reacciones físicas } \\
\hline Inquietud $^{2}$ & $33,2 \%$ & $41,9 \%$ & $24,9 \%$ \\
\hline Sentimientos de depresión y tristeza & $48,6 \%$ & $34,9 \%$ & $16,5 \%$ \\
\hline Ansiedad, angustia o desesperación. & $41,1 \%$ & $39,5 \%$ & $19,5 \%$ \\
\hline Problemas de concentración. & $35,9 \%$ & $40,3 \%$ & $23,8 \%$ \\
\hline Sentimientos de agresividad o aumento de irritabilidad & $68,1 \%$ & $20,8 \%$ & $11,1 \%$ \\
\hline \multicolumn{4}{|l|}{ Reacciones psicológicas } \\
\hline Trastornos del sueño ${ }^{3}$ & $56,8 \%$ & $29,2 \%$ & $14,1 \%$ \\
\hline Fatiga crónica ${ }^{4}$ & $55,1 \%$ & $24,1 \%$ & $20,8 \%$ \\
\hline Dolores de cabeza o migrañas. & $50,3 \%$ & $27,6 \%$ & $22,2 \%$ \\
\hline Problemas de digestión, dolor abdominal o diarrea ${ }^{5}$ & $73,0 \%$ & $15,1 \%$ & $11,9 \%$ \\
\hline Rascarse, morderse las uñas, frotarse, etc. & $65,4 \%$ & $19,2 \%$ & $15,4 \%$ \\
\hline Somnolencia o mayor necesidad de dormir & $40,0 \%$ & $32,4 \%$ & $27,6 \%$ \\
\hline \multicolumn{4}{|l|}{ Reacciones comportamentales } \\
\hline Conflictos o tendencia a polemizar o discutir & $67,8 \%$ & $23,2 \%$ & $8,9 \%$ \\
\hline Aislamiento de los demás & $63,8 \%$ & $25,1 \%$ & $11,1 \%$ \\
\hline Desgano para realizar las labores escolares & $52,4 \%$ & $36,5 \%$ & $11,1 \%$ \\
\hline Aumento o reducción del consumo de alimentos ${ }^{6}$ & $45,9 \%$ & $33,0 \%$ & $21,1 \%$ \\
\hline \multicolumn{4}{|l|}{ Estrategias de afrontamiento } \\
\hline Habilidad asertiva & $15,9 \%$ & $32,4 \%$ & $51,4 \%$ \\
\hline Elaboración de un plan y ejecución de sus tareas & $20,8 \%$ & $37,3 \%$ & $41,9 \%$ \\
\hline Elogios a sí mismo ${ }^{7}$ & $44,6 \%$ & $32,4 \%$ & $23,0 \%$ \\
\hline La religiosidad $^{8}$ & $55,1 \%$ & $22,7 \%$ & $22,2 \%$ \\
\hline Búsqueda de información sobre la situación ${ }^{9}$ & $38,9 \%$ & $34,6 \%$ & $26,2 \%$ \\
\hline Ventilación y confidencias & $43,0 \%$ & $32,7 \%$ & $24,1 \%$ \\
\hline Uso de psicofármacos & $92,2 \%$ & $6,5 \%$ & $1,4 \%$ \\
\hline
\end{tabular}

${ }^{1}$ Este ítem presentó diferencias según la ocupación $\left(\chi^{2}=12,35 p=0,015\right)$ para $p \leq 0,05$

${ }^{2}$ Este ítem presentó diferencias según la edad $\left(\chi^{2}=9,98 p=0,041\right)$ para $p \leq 0,05$

${ }^{3}$ Este ítem presentó diferencias según el semestre $\left(\chi^{2}=9,76 p=0,045\right)$ para $p \leq 0,05$

${ }^{4}$ Este ítem presentó diferencias según el estrato socioeconómico $\left(\chi^{2}=10,14 p=0,038\right)$ para $p \leq 0,05$

${ }^{5}$ Este ítem presentó diferencias según la ocupación $\left(\chi^{2}=12,54 p=0,014\right)$ para $p \leq 0,05$

${ }^{6}$ Este ítem presentó diferencias según el semestre $\left(\chi^{2}=11,63 p=0,020\right)$ para $p \leq 0,05$

${ }^{7}$ Este ítem presentó diferencias según la edad $\left(\chi^{2}=10,42 p=0,034\right)$ para $p \leq 0,05$

${ }^{8}$ Este ítem presentó diferencias según el estrato socioeconómico $\left(\chi^{2}=10,13 p=0,038\right)$ para $p \leq 0,05$

${ }^{9}$ Este ítem presentó diferencias según la institución $\left(\chi^{2}=12,1 p=0,007\right)$ para $p \leq 0,05$ 


\section{Discusión}

En los resultados, la categoría alta, media y baja hicieron referencia a la frecuencia en la que el contenido descrito en cada ítem producía estrés en el estudiante. Los resultados, en su mayor porcentaje, están entre el nivel medio y bajo. Ninguno de los ítems tuvo su mayor porcentaje en la categoría alta. A pesar de esto, sí hay algunos porcentajes que resultan de interés. Por ejemplo, la sobrecarga de tareas y trabajos escolares, el tiempo limitado para hacer los trabajos y el tipo de trabajos que asignan los profesores fueron, en este orden, las tres situaciones y condiciones que con mayor frecuencia generaron estrés en los estudiantes. La inquietud y los problemas de concentración fueron las reacciones físicas que con mayor frecuencia se asociaron con el estrés. Respecto a las reacciones psicológicas, la somnolencia, los dolores de cabeza y la fatiga crónica fueron los síntomas de estrés más frecuentes según el reporte de los estudiantes. El aumento o reducción del consumo de alimentos fue la reacción comportamental de mayor frecuencia. Finalmente, las estrategias de afrontamiento más frecuentemente utilizadas por los estudiantes fueron la habilidad asertiva y la elaboración de plan y la ejecución de tareas. Estos hallazgos coinciden, en general, con los reportados por la literatura (Toribio-Ferrera y Franco-Bárcenas, 2016).

Para la categoría Situaciones y condiciones estresantes, el ítem con más porcentaje en la clasificación alto fue el de Sobrecarga de tareas y trabajos escolares. De acuerdo con Berrio y Mazo (2011), los estudiantes refieren experimentar altos niveles de estrés académico debido a las altas responsabilidades de actividades programadas, como son los trabajos escritos, exposiciones, evaluaciones, entre otras. Además, existen otros factores que pueden coexistir al estrés que generan las responsabilidades en el estudio como son la asistencia a clase, las percepciones y actitudes sobre las diferentes metodologías de aprendizaje y enseñanza, la percepción de logro, la satisfacción con la carrera y la institución académica, el sistema relacional en el aula de clases y la vida académica en general. Sumado a la lista, Pozos, Preciado, Plascencia, Acosta y Aguilera (2015) identifican otros estresores como la necesidad de participar en clase, responder a los compromisos en las tutorías, las actividades en grupo, exposiciones y sustentaciones orales, y la rivalidad entre los mismos compañeros. La ansiedad ante los exámenes también es un factor relevante (Álvarez, Aguilar y Lorenzo, 2012).

La sobrecarga de tareas y trabajos escolares y el tiempo limitado para hacer los trabajos han sido reportados en otros estudios nacionales e internacionales como los mayores estresores académicos (Barraza, 2003; Bedoya-Lau, 
Matos y Zelaya, 2014; Berrío y Mazo, 2011; Castillo et al. 2016, GarcíaRos, Pérez-González, Pérez-Blasco y Natividad, 2012; Martín, 2007). El estrés psicológico surge cuando, en la valoración cognitiva sobre un acontecimiento, los recursos para afrontarlo son inferiores a las demandas de la situación (Lazarus \& Folkman, 1986). En el contexto universitario, los niveles de lectura y la cantidad de tareas aumentan y son una queja constante de los estudiantes quienes, en algunos casos quizás debido a deficiencias académicas heredadas de la educación media (menor exigencia, inapropiados hábitos de estudio, desmotivación escolar, entre otras), los perciben como desbordantes y se angustian al considerar las consecuencias que habría si no responden satisfactoriamente a esas nuevas demandas.

Ni la sobrecarga de tareas y trabajos escolares, ni el tiempo limitado para hacer los trabajos presentaron diferencias estadísticamente significativas al compararlas en función del sexo, la edad, la ocupación, el semestre, el estrato socioeconómico o el tipo de institución. De tal forma que los niveles de estrés académico de estas dos condiciones son similares al compararlos en función de esas variables. Esta estabilidad e independencia es relevante porque demuestra, en cierta medida, que el efecto estresor podría depender más de la situación en sí misma, que de los recursos y capacidades de afrontamiento de los estudiantes (Sarubbi De Rearte y Castaldo, 2013). En otras palabras, al no hallar diferencias en los niveles de estrés para estas dos condiciones en función de todas esas variables, podría entenderse que el estrés depende más de una condición objetiva (la naturaleza del estímulo) y no tanto subjetiva (la valoración cognitiva individual sobre ese estímulo).

Así, si se hubiesen encontrado diferencias en función del semestre y, por ejemplo, los estudiantes de primeros semestres hubiesen presentado mayores niveles de estrés académico en comparación con los de semestres intermedios, entonces podría atribuírsele la diferencia en el nivel de estrés a la diferencia en la capacidad de los estudiantes para afrontar esta situación, siendo mejor la capacidad del segundo grupo (por razones que habría que plantear). Sin embargo, lo que estos resultados sugieren es que la sobrecarga de tareas y trabajos escolares y el tiempo limitado para hacer los trabajos es una condición que genera estrés con independencia del semestre (y de las otras variables consideradas) y, por tal razón, debería ser evaluada atentamente esa condición. ¿Están las materias o asignaturas de los diferentes programas académicos diseñadas para formar al estudiante en lo relevante? ¿O acaso están saturadas de contenidos e información que en su mayoría termina por ser olvidada por el estudiante al semestre siguiente? ¿Habría que pensar más en la calidad del contenido y menos en la cantidad del mismo? 
Incluso algunos profesores se angustian ante programas de curso tan extensos y se ven obligados a realizar acrobacias pedagógicas y malabares metodológicos para cumplirlos a cabalidad. Pero, ¿̇cómo enseñar y aprender en esta era de la información? La pregunta no se limita a un asunto pedagógico, sino que se expande hacia un asunto de salud. Porque varios estudios han puesto en evidencia la relación del estrés académico con alteraciones en la salud física y mental de los estudiantes (Castillo et al., 2016; Gutiérrez et al., 2010; Montoya et al., 2010; Parra-Osorio et al., 2015; Pozos-Radillo et al., 2014; Restrepo et al., 2018). Los hallazgos sobre los efectos físicos, psicológicos y comportamentales del estrés académico en esta muestra de estudiantes sirven para constatar que hay un conjunto relevante de reacciones de este tipo que deben ser consideradas debido al malestar inmediato que produce en los estudiantes y por las eventuales consecuencias que podrían aparecer con el tiempo (Castrillón, Sarsosa, Moreno y Moreno, 2105).

La inquietud, los problemas de concentración, la somnolencia, los dolores de cabeza, la fatiga crónica, y el aumento o reducción del consumo de alimentos fueron las reacciones de mayor frecuencia asociadas con el estrés académico en los estudiantes, en la categoría de Reacciones físicas (Águila, Calcines, Monteagudo y Nieves, 2015). La inquietud presentó diferencias estadísticamente significativas en función de la edad. De acuerdo con los resultados de los análisis, los niveles más altos de inquietud se presentaron en el grupo de 18 a 19 años, seguido del grupo de 20 a 21 años. Estos grupos de edad corresponden a los estudiantes de semestres iniciales e intermedios. Los trastornos del sueño también presentaron diferencias estadísticamente significativas, pero en función del semestre. Nuevamente, durante los primeros semestres (1-3) y los semestres intermedios (4-6) se reportaron los niveles más altos de trastornos de sueño. Durante estos mismos semestres se encontraron diferencias estadísticamente significativas en cuanto al aumento o reducción del consumo de alimentos. La fatiga crónica presentó diferencias estadísticamente significativas en función del estrato socioeconómico. En los estratos 1 y 2 se presentaron los niveles más altos de esta condición de salud.

Para la categoría Reacciones comportamentales, el ítem con más porcentaje en la clasificación alto fue el de Aumento o reducción del consumo de alimentos (este ítem, además, presentó diferencias según el semestre $(\chi 2=11,63 p=$ $0,020)$ para $p \leq 0,05$ ), en lo cual se ha demostrado que el estrés académico conlleva a modificaciones graves en el estilo de vida, específicamente en los hábitos de alimentación saludables y al aumento del consumo de alcohol, cigarrillo o café y al incremento del sedentarismo que afectan la salud, el rendimiento intelectual y la calidad de vida (Hystad, Eid, Laberg, Johnsen \& 
Bartone, 2009; Lara, Saldaña, Fernández y Delgadillo, 2015). Sumado a esto, se ha encontrado que los niveles de estrés están relacionados con el semestre que se cursa durante la carrera. Dos de los picos que se resaltan son cuarto y noveno semestre, pues en el primero se inicia con el saber propio de la carrera en curso y en el segundo se proyecta el período de prácticas, la tesis de grado y la graduación (Mazo et al., 2013).

Sobre esto, Calais, Carrara, Brum, Batista, Yamada y Oliveira (2007) advierten la diferencia del estrés académico en los estudiantes de los primeros semestres en los que está ligado al incremento de las responsabilidades y a la percepción de mayor dificultad en las tareas en la medida que se avanza en la carrera. Mientras que, en los estudiantes de últimos semestres, el estrés está ligado a la perspectiva de desempeño a nivel profesional. Agregan que en los estudiantes de primer semestre prevalecen los síntomas físicos y en los últimos semestres, los síntomas de tipo psicológico. Puede agregarse que, durante el período de exámenes parciales y finales, se incrementa el consumo de sustancias psicoactivas, la ingestión de tranquilizantes que repercuten en problemas de salud.

Martínez y Díaz (2007) refieren que el estrés puede ser visto a través de una gama de manifestaciones físicas, como son la inquietud, el nerviosismo, la tensión y el cansancio. Relacionado con esto, el estrés de tipo académico ha estado relacionado con dolores de cabeza de manera frecuente, mareos, problemas digestivos, problemas de sueño, somnolencia o mayor necesidad de dormir. Esto se evidencia en la muestra de estudiantes evaluados durante la presente investigación ya que, de acuerdo a los resultados en la categoría de Reacciones psicológicas, un alto porcentaje presenta inquietud o lo que es lo mismo, incapacidad de relajarse y estar tranquilo, de concentrarse o disponerse de manera adecuada a la realización de las tareas pertinentes.

El modelo cognitivo-transaccional establece que las estrategias de afrontamiento corresponden a un curso dinámico desde lo cognitivo hacia lo conductual que le permiten al individuo enfrentarse a las demandas, internas y externas, y evitar el desequilibrio tanto psicológico como fisiológico. En general, las estrategias de afrontamiento implican la autorregulación frente a las percepciones de estrés y sus desencadenantes (Compas, Connor-Smith, Saltzman, Thomsen \& Wadsworth, 2001). En cuanto a las principales estrategias de afrontamiento reseñadas por algunos autores están aquellas en las que el sujeto reevalúa positivamente la experiencia y reduce los aspectos negativos de la misma; así como aquellas en las que el sujeto busca apoyo social tanto emocional como instrumental. Las primeras se refieren a la ayuda que pueda contribuir al alivio y comprensión desde sus propias cogniciones y 
emociones. Las segundas, a la búsqueda de nueva información que permita la solución del problema (Cabanach, Valle, Rodríguez, Piñeiro y Freire, 2010). Adicionalmente, hay estrategias que le permiten a la persona llevar a cabo acciones analíticas, racionales, para cambiar la situación que representa la problemática.

De acuerdo con los resultados, la estrategia de afrontamiento que más se utiliza es la habilidad asertiva, que tiene que ver con la capacidad de defender las preferencias, ideas o sentimientos sin dañar a nadie $(51,4 \%)$, seguida de elaboración de un plan y ejecución de sus tareas (41,9\%). Los resultados coinciden con el estudio llevado a cabo en la Universidad Nacional Mayor de San Marcos, Perú, con estudiantes universitarios de odontología, en la que se muestran frecuencias altas de los estudiantes en habilidad asertiva $(51,6 \%)$, seguido de la elaboración de un plan $(38,7 \%)$ (Chiyong y Villacampa, 2015). Este último asunto es importante, y es de resaltar, puesto que puede interpretarse que el estudiante es quien descubre que una manera de combatir el estrés es apropiarse y defender lo que considera correcto y que logra desarrollar a través de su propio aprendizaje y del cumplimiento de las diferentes actividades.

Este resultado también coincide con los hallazgos de Nieves, Satchimo, González y Jiménez (2013), quienes en su estudio sobre niveles de estrés académico en estudiantes universitarios, encontraron que las estrategias de afrontamiento mayormente empleadas fueron: la habilidad asertiva (defender nuestras preferencias, ideas o sentimientos sin dañar a otros), con una incidencia en el 40.90\% de los alumnos evaluados, y la elaboración de un plan y ejecución de sus tareas, que tuvo una puntuación del $35.75 \%$. Peña, Bernal, Pérez, Ávila y García (2018) evaluaron en su investigación la frecuencia e intensidad del estrés e identificaron las estrategias que utilizaban los estudiantes universitarios para afrontarlo, encontrando también que la estrategia más utilizada según el SISCO fue la habilidad asertiva con un $13.3 \%$.

Así mismo, Barraza (2008), encontró que las estrategias de afrontamiento que utilizan con mayor frecuencia los alumnos de las maestrías en Educación fueron: la elaboración de un plan y ejecución de sus tareas, mientras que las que utilizan con menos frecuencia son: los elogios a sí mismo y la religiosidad, lo cual es semejante a los resultados que arrojan este estudio. En la investigación de Castillo, Barrios y Alvis (2018), llevada a cabo con estudiantes de enfermería en varias universidades de Cartagena, también se encontró, que la estrategia que menos utilizó la muestra evaluada fue los elogios hacia sí mismos, con una puntuación del 21,2\%; resultado que es similar al de esta investigación, ya que la variable tuvo una frecuencia clasificada como alta en 
el 23,0\% de la población. Ticona, Paucar y Llerena (2010), encontraron también que la estrategia menos empleada por los estudiantes para hacer frente al estrés académico fue la religión (7,52\%)

Con relación a la estrategia de afrontamiento ventilación y confidencias (verbalización de la situación que le preocupa), los hallazgos de Nieves et al. (2013), son similares a los de la presente investigación, pues reportaron que el $25.75 \%$ de la muestra expresó implementarla casi siempre o siempre, y en este estudio se obtuvo una puntuación del $24.1 \%$ de la población que reportó utilizarla con alta frecuencia. Con respecto a la utilización de psicofármacos, los resultados de este estudio coinciden también con los de los autores en mención, al concluir que un gran porcentaje de la población evaluada, reporta que no consume psicofármacos (85\%), mientras en esta investigación el $92,2 \%$ de los encuestados, refiere no emplearlos.

Ahora bien, Casuso (2011), señala que dentro de las variables moduladoras del estrés académico, la percepción de autoeficacia de los estudiantes, es descrita como factor clave tanto en la aparición de respuestas de estrés, como en el proceso valorativo y de afrontamiento del mismo, por lo cual es posible que aquellos alumnos con mayores niveles de autoeficacia, en comparación con los que se perciben como menos eficaces, generen expectativas más elevadas sobre su desempeño, puesto que tienen mayor confianza en sus capacidades y valoran el entorno académico como menos amenazante, lo que permite que afronten las situaciones con más probabilidad de adaptarse con éxito a las demandas académicas y que experimenten mejores niveles de bienestar general, lo cual coincide con los resultados de la presente investigación, ya que las puntuaciones más altas obtenidas para la población, con relación a las estrategias que emplean para hacer frente al estrés académico, son la habilidad asertiva y la elaboración de un plan y ejecución de sus tareas, lo que quiere decir que su estilo de afrontamiento se encuentra centrado en el problema.

De acuerdo a Casuso (2011), los jóvenes universitarios por lo general buscan el apoyo social y usan estrategias de confrontación para solucionar el problema, para que así las situaciones problemáticas no influyan negativamente en su rendimiento académico, lo cual apoya los resultados arrojados por este estudio, con relación a que los estudiantes evaluados, usan con mayor frecuencia un afrontamiento centrado en el problema y hacen menor uso de estrategias centradas en la emoción o en la evitación, como es el caso de la religiosidad y el uso de psicofármacos. Otra de las investigaciones que apoya los resultados arrojados, es la de Palacio, Caballero, González, Gravini y Contreras (2012) quienes stablecieron que los estudiantes universitarios tenían bajos niveles de burnout académico, por lo cual concluían que emplea- 
ban estrategias de afrontamiento orientadas al problema y éstas les permitía mantener su atención en los estudios, sin afectar su desempeño. De igual modo, Sicre y Casaro (2014), concluyeron que la estrategia predominantemente utilizada por los estudiantes universitarios era el afrontamiento por aproximación cognitiva: los esfuerzos cognitivos para entender y prepararse para enfrentar un elemento generador de estrés y sus consecuencias.

Por otro lado, dichos resultados se diferencian de los mostrados en la investigación de Ortiz, Tafoya, Farfán y Jaimes (2013), quienes observaron que la estrategia más utilizada fue la de búsqueda de apoyo social que presentó una disminución para el segundo período del año. En cuanto a las menos utilizadas, los resultados coinciden en las estrategias de afrontamiento de religiosidad y elogios a sí mismo. A manera de explicación, se puede concluir que los resultados contradictorios, y otros similares, pueden comprenderse desde modelo de Lazarus \& Folkman (1986) quienes terminan expresando que no todas las personas reaccionan de forma similar frente a las mismas fuentes que generan estrés, pues el estímulo será percibido de forma positiva o de manera negativa, sumado a las estrategias de afrontamiento individual de los estudiantes que les permitirá hacer frente a la situación estresante.

Un asunto clave a discutir con base en los resultados descritos anteriormente es que no necesariamente las estrategias de afrontamiento que más usan los estudiantes frente al estrés bajo las exigencias en la universidad son las más eficaces. Además, que se hace complejo determinar criterios para identificar que una estrategia es más eficaz que otra. Sobre esto último, Somerfield \& McCrae (2000) no dudaron en señalar la dificultad que se tiene para llegar a concluir si una estrategia es mejor que las demás, puesto que esta depende realmente del contexto específico, del problema o de la situación estresante y de las necesidades que se tienen en cada escenario y lo que más conviene como respuesta esperable en dicha situación. En el estudio de Chiyong \& Villacampa, la habilidad asertiva le permitió afrontar efectivamente las reacciones del estrés a menos del $50 \%$ de los estudiantes.

\section{Conclusiones}

En conjunto, todas las anteriores reacciones físicas, psicológicas y comportamentales reportadas por esta muestra de estudiantes universitarios obligan a situar en un lugar de interés la discusión sobre la salud mental y física de esta población. Desde una perspectiva educativa, este interés está justificado puesto que ese conjunto de reacciones no hará más que menguar y perjudicar la capacidad intelectual y el desempeño académico de los estudiantes y, en 
muchos casos, el estrés académico (en conjunción con otras problemáticas) podría derivar en fracaso y deserción. Los íntimos vínculos entre el estrés académico, la ansiedad, la depresión, el consumo de sustancias y la ideación suicida podrían entretejer una trampa muy riesgosa para la salud y el proyecto de vida de personas jóvenes, en su mayoría menores de 25 años.

De acuerdo con Muñoz (2004), son insuficientes las investigaciones donde se abordan de manera rigurosa el impacto que tiene el sistema educativo universitario en los estudiantes versus la demanda académica y las actividades implicadas en las situaciones vividas allí y que podrían repercutir negativamente en la salud, el bienestar y el rendimiento del estudiante. Considerando que el ingreso a la universidad es de por sí un factor importante de estrés, es relevante deliberar sobre algunas medidas preventivas para el estrés académico, en tanto es un factor de riesgo para el bajo rendimiento escolar (Jerez \& Oyarzo, 2015). Si bien son importantes algunos de los aportes investigativos alrededor de la evaluación del estrés académico, y tomando como referencia los resultados, es importante que los estudiantes identifiquen factores causales del estrés que permitan prevenir aquellos efectos negativos que pueden incidir en la formación, aprendizaje y rendimiento académico (Zárate et al., 2017).

\section{Referencias}

Águila, B. A., Calcines, M., Monteagudo, R. y Nieves, Z. (2015). Estrés académico. Edumecentro, 7(2), 163-178. http://scielo.sld.cu/scielo.php?script=sci_arttext\&p $\mathrm{id}=\mathrm{S} 2077-28742015000200013$

Álvarez, J., Aguilar, J. M. y Lorenzo, J. J. (2012). La Ansiedad ante los Exámenes en Estudiantes Universitarios: Relaciones con variables personales y académicas. Electronic Journal of Research in Educational Psychology, 10(1), 333-354. https:// www.redalyc.org/articulo.oa?id $=293123551017$

Awé, C., Gaither, C. A., Crawford, S. Y., \& Tieman, J. (2016). A Comparative Analysis of Perceptions of Pharmacy Students' Stress and Stressors across Two Multicampus Universities. American Journal of Pharmaceutical Education, 80(5), 82. https:// www.ncbi.nlm.nih.gov/pmc/articles/PMC4937977/

Barraza, A. (2003). El estrés académico de los alumnos de Educación Media Superior, Hermosillo. Memoria VIII Congreso Nacional de Investigación Educativa. https://www. google.com/url? sa $=$ t\&rct $=j \& q=\& e s r c=s \&$ source $=$ web\&cd $=1 \& v e d=2$ ahUKEwiYnr6Rx8DpAhXImuAKHZoWBccQFjAAegQIBBAB\&url=https\%3A\%2F\%2Fdi- 
alnet.unirioja.es\%2Fdescarga\%2Farticulo\%2F2358918.pdf\&usg=AOvVaw2raJjoAP1o6zYYgqr0Rfd

Barraza, A. (2007). El Inventario SISCO del Estrés Académico. Investigación Educativa Duranguense, 7, 90-93. https://dialnet.unirioja.es/servlet/articulo?codi$g_{0}=2358921$

Barraza, A. (2007a). Propiedades psicométricas del Inventario SISCO del Estrés Académico. Revista Electrónica Psicologíacientífica.com. Disponible en: http:// www.psicologiacientifica.com/sisco-propiedades-psicometricas/

Barraza, A. (2008). El estrés académico en alumnos de maestría y sus variables moduladoras: un diseño de diferencia de grupos. Avances en Psicología Latinoamericana, 26(2), 270-289. https://revistas.urosario.edu.co/index.php/ apl/article/view/67

Bedoya-Lau, F., Matos, L., y Zelaya, E. (2014). Niveles de estrés académico, manifestaciones psicosomáticas y estrategias de afrontamiento en alumnos de la facultad de medicina de una universidad privada de Lima en el año 2012. Revista de Neuropsiquiatría, 77(4), 262-270. http://www.scielo.org.pe/pdf/rnp/v77n4/ a09v77n4.pdf

Bermúdez, S. B., Durán, M., Escobar, C., Morales, A., Monroy, S. A., Ramírez, A., Ramírez, J., Trejos, J. L., Castaño, J. J. y González, S. P. (2006). Evaluación de la relación entre rendimiento académico y estrés en estudiantes de Medicina. MedUNAB, 9(3), 198-205. https://revistas.unab.edu.co/index.php/medunab/ article/view/135

Berrío, N. y Mazo, R. (2011). Estrés Académico. Revista de Psicología Universidad de Antioquia, 3(2), 65-82. https://aprendeenlinea.udea.edu.co/revistas/index.php/ psicologia/article/view/11369

Cabanach, R. G., Valle, A., Rodríguez, S., Piñeiro, I., y Freire, C. (2010). Escala de afrontamiento del estrés académico (A-CEA). Revista Iberoamericana de Psicología y Salud, 1(1), 51-64. https://www.redalyc.org/pdf/2451/245116411005.pdf

Calais, S. L., Carrara, K., Brum, M. M., Batista, K., Yamada, J. K., y Oliveira, J. R. S. (2007). Stress entre calouros e veteranos de jornalismo. Estudos de Psicologia (Campinas), 24(1), 69-77. http://www.scielo.br/scielo.php?script=sci arttext\&pid $=$ S0103-166X2007000100008

Caldera, J. F., Pulido, B. E. y Martínez, M. G. (2007). Niveles de estrés y rendimiento académico en estudiantes de la carrera de Psicología del Centro Universitario de Los Altos. Revista de Educación y Desarrollo, 7, 77-82. http://www.cucs.udg.mx/ revistas/edu_desarrollo/anteriores/7/007_Caldera.pdf 
Castillo, C., Chacón de la Cruz, T. y Díaz-Véliz, G. (2016). Ansiedad y fuentes de estrés académico en estudiantes de carreras de la salud. Investigación en Educación Médica, 5(20), 230-237. https://www.sciencedirect.com/science/article/pii/ S2007505716000491

Castillo, I., Barrios, A. y Alvis, L. (2018). Estrés académico en estudiantes de enfermería de Cartagena, Colombia. Investigación en Enfermería: Imagen y Desarrollo, 20(2). https://revistas.javeriana.edu.co/files-articulos/IE/20-2\%20 (2018-II)/145256681002/

Castrillón, E., Sarsosa, K., Moreno, F. y Moreno S. (2105). Estrés académico y sus manifestaciones inmunológicas: La evidencia de la psico-neuroendocrinoinmunología. Salutem Scientia Spiritus, 1(1), 16-28. https://revistas.javerianacali. edu.co/index.php/salutemscientiaspiritus/article/view/1358

Casuso, M. J. (2011). Estudio del estrés, engagement y rendimiento académico en estudiantes universitarios. Tesis doctoral. Facultad de Enfermería, Fisioterapia, Podología y Terapia Ocupacional. España: Universidad de Málaga. https:// riuma.uma.es/xmlui/bitstream/handle/10630/4926/TD\%20Maria\%20Jesus\%20 Casuso\%20Holgado.pdf? sequence $=1$

Chiyong, T. y Villacampa S. (2015) Estrés y desempeño académico en estudiantes de Odontología. Odontología Sanmarquina, 18(1), 23-27. https:// revistasinvestigacion.unmsm.edu.pe/index.php/odont/article/view/11336

Compas, B. E., Connor-Smith, J. K., Saltzman, H., Thomsen, A. H. \& Wadsworth, M. E. (2001). Coping with stress during childhood and adolescence: problems, progress, and potential in theory and research. Psychological Bulletin, 127(1), 87127. https://www.ncbi.nlm.nih.gov/pubmed/11271757

Díaz, M. Y., Ortigosa, F. E., Díaz, C. A., y Castillo, E. Y. (2015). Intervención psicoeducativa para disminuir el estrés académico en estudiantes de primer año de medicina. Revista electrónica Dr. Zoilo E. Marinello Vidaurreya, 40(5), 1-13. http://revzoilomarinello.sld.cu/index.php/zmv/article/view/65

Domínguez, R. A., Guerrero, G. J., y Domínguez, J. G. (2015). Influencia del estrés en el rendimiento académico de un grupo de estudiantes universitarios. Educación y ciencia, 4(43), 31-40. http://www.educacionyciencia.org/index.php/ educacionyciencia/article/view/313

Elias, H., Ping, W. S. \& Abdullah, M. C. (2011). Stress and academic achievement among undergraduate students in Universiti Putra Malaysia. Procedia - Social and Behavioral Sciences, 29, 646-655. https://www.sciencedirect.com/science/ article/pii/S1877042811027492 
Feldman, L., Goncalves, L., Chacón-Puignau, G., Zaragoza, J., Bagés, N. y De Pablo, J. (2008). Relaciones entre estrés académico, apoyo social, salud mental y rendimiento académico en estudiantes universitarios venezolanos. Universitas Psychologica, 3(7), 739-751. https://revistas.javeriana.edu.co/index.php/ revPsycho/article/view/406

Fernández-Hileman, M. D. R., Corengia, A. y Durand, J. (2014). Deserción y retención universitaria: una discusión bibliográfica. Pensando Psicología, 10(17), 85-96. https://revistas.ucc.edu.co/index.php/pe/article/view/787

García-Ros, R., Pérez-González, F., Pérez-Blasco, J. y Natividad, L. A. (2012). Evaluación del estrés académico en estudiantes de nueva incorporación a la universidad. Revista Latinoamericana de Psicología, 44(2), 143-154. https://www. redalyc.org/articulo.oa?id $=80524058011$

Gutiérrez, J. A., Montoya, L. P., Toro, B. E., Briñón, M. A., Rosas, E. y Salazar, L. E. (2010). Depresión en estudiantes universitarios y su asociación con el estrés académico. CES Medicina, 24(1), 7-17. http://revistas.ces.edu.co/index.php/ medicina/article/view/1011

Hystad, S. W., Eid, J., Laberg, J. C., Johnsen, B. H., \& Bartone, P. T. (2009). Academic Stress and Health: Exploring the Moderating Role of Personality Hardiness. Scandinavian. Journal of Educational Research, 53(5), 421-429. https://www. tandfonline.com/doi/abs/10.1080/00313830903180349

Jerez, M. M. y Oyarzo, B. C. (2015). Estrés académico en estudiantes del Departamento de Salud de la Universidad de Los Lagos Osorno. Revista Chilena de Neuropsiquiatría, 53(3), 149-157. https://scielo.conicyt.cl/scielo.php?script=sci arttext\&pid $=$ S0717-92272015000300002

Labrador, C. C. (2012). Estrés académico en estudiantes de la facultad de farmacia y bioanálisis.UniversidaddelosAndes. Mérida-Venezuela. (Tesisdoctoral).Universidad de Alcalá, Venezuela. https://ebuah.uah.es/dspace/handle/10017/16001

Lara, N., Saldaña, Y., Fernández, N. y Delgadillo, H. J. (2015). Salud, calidad de vida y entorno universitario en estudiantes mexicanos de una universidad pública. Hacia la Promoción de la Salud, 20(2), 102-117. http://www.scielo.org.co/pdf/ hpsal/v20n2/v20n2a08.pdf

Lazarus, R. \& Folkman, S. (1986). Estrés y procesos cognitivos. Barcelona: Martínez Roca.

Malo, D., Guerty, S., Cáceres, C. y Peña, G. H. (2010). Validación del inventario SISCO del estrés académico y análisis comparativo en adultos jóvenes de la Universidad Industrial de Santander y la Universidad Pontificia Bolivariana, Seccional Bucaramanga, Colombia. Revista Electrónica de la Red Durango de 
Investigadores Educativos A C, 2(3), 26-42. https://dialnet.unirioja.es/servlet/ articulo? codigo $=6534519$

Martín, I. M. (2007). Estrés académico en estudiantes universitarios. Apuntes de Psicología, 25(1), 87-99. http://copao.cop.es/files/contenidos/VOL25_1_7.pdf

Martínez, E.S. y Díaz, D.A. (2007). Una aproximación psicosocial al estrés escolar. Educación y Educadores, 2(10), 11-22. https://educacionyeducadores.unisabana. edu.co/index.php/eye/article/view/687

Mazo, R., Londoño, K. y Gutiérrez, Y, F. (2013). Niveles de estrés académico en estudiantes universitarios. Informes Psicológicos, 13(2), 121-134. https://revistas. upb.edu.co/index.php/informespsicologicos/article/view/2796/2441

Montoya, L. M., Gutiérrez, J. A., Toro, B. E., Briñón, M. A., Rosas, E. y Salazar, L. E. (2010). Depresión en estudiantes universitarios y su asociación con el estrés académico. Revista CES Medicina, 24(1), 7-17. http://revistas.ces.edu.co/index. $\mathrm{php} /$ medicina/article/view/1011/735

Muñoz, F. J. (2004). El estrés académico. Problemas y soluciones desde una perspectiva psicosocial. Huelva: Universidad de Huelva Publicaciones.

Nieves, Z., Satchimo, A., González, Y. y Jiménez, M. (2013). Algunas consideraciones acerca del estrés académico en los estudiantes universitarios. Revista Psicoespacios, 7(11), 91-116. http://revistas.iue.edu.co/revistasiue/index.php/Psicoespacios/ article/view/215

Ortiz, S., Tafoya, S., Farfán, A. y Jaimes, A. (2013). Rendimiento Académico, Estrés y Estrategias de Afrontamiento en Alumnos del Programa de Alta Exigencia Académica de la Carrera de Medicina. Revista Med, 21(1), 29-37. https://revistas. unimilitar.edu.co/index.php/rmed/article/view/1150

Palacio, J., Caballero, C., González, O., Gravini, M. y Contreras, K. (2012). Relación del burnout y las estrategias de afrontamiento con el promedio académico en estudiantes universitarios. Universitas Psychologica, 11(2), 535-54. https://www. redalyc.org/articulo.oa?id=64723241015

Parra-Osorio, L., Rojas-Castaño, L., Patiño-Córdoba, I., Caicedo-Ausecha, G. y Valderrama-Aguirre, A. (2015). Correlación en Síndrome de Burnout y Estrés Académico en Estudiantes de Medicina de una Facultad de Salud en Cali, Colombia. Revista Colombiana de Salud Ocupacional, 5(3), 31-34. https://revistas. unilibre.edu.co/index.php/rc_salud_ocupa/article/view/4917

Peña, E., Bernal, L., Pérez, R., Ávila, L. y García, K. (2018). Estrés y estrategias de afrontamiento en Estudiantes de Nivel Superior de la Universidad Autónoma de Guerrero. NURE investigación, 15(92), 1-8. https://dialnet.unirioja.es/servlet/ articulo? codigo $=6279759$ 
Peñacoba, C. y Moreno-Jiménez, B. (1999). La escala de estresores universitarios (EEU) Una propuesta para la evaluación del estrés en grupos poblacionales específicos. Ansiedad y Estrés, 5(1), 61-78. https://dialnet.unirioja.es/servlet/ articulo? codigo $=186719$

Pozos, B., Preciado, M., Plascencia, A., Acosta, M., y Aguilera, M. (2015). Estrés académico y síntomas físicos, psicológicos y comportamentales en estudiantes mexicanos de una Universidad pública. Ansiedad y Estrés, 21(1), 35-42. https:// dialnet.unirioja.es/servlet/revista? codigo $=97$

Pozos-Radillo, B. E., Preciado-Serrano, M., Acosta-Fernández, M., Aguilera-Velasco, M. y Delgado-García, D. D. (2014). Academic stress as a predictor of chronic stress in university students. Psicología Educativa, 20(1), 47-52. https://www. sciencedirect.com/science/article/pii/S1135755X14000074

Pulido, M. A., Serrano, M. L., Valdés, E., Chávez, M. T., Hidalgo, P. y Vera, F. (2011). Estrés académico en estudiantes universitarios. Psicología y Salud, 21(1), 31-37. http://copao.cop.es/files/contenidos/VOL25_1_7.pdf

Reddy, K. J., Menon K. R. \& Thattil A. (2018). Academic Stress and its Sources Among University Students. Biomedical and Pharmacology Journal, 11(1), 531-537. http://biomedpharmajournal.org/vol11no1/academic-stress-and-its-sourcesamong-university-students/

Restrepo, J. E., Sánchez, O. A., Calderon, G., Castañeda, T., Osorio, Y. y Diez, P. (2018). Depresión y su relación con el consumo de sustancias psicoactivas, el estrés académico y la ideación suicida en estudiantes universitarios colombianos. Salud y Drogas, 18(2), 227-239. https://dialnet.unirioja.es/servlet/ articulo codigo $=6546342$

Rosales, J. G. (2016). Estrés académico y hábitos de estudio en universitarios de la Carrera de Psicología de un Centro de Formación Superior Privada de Lima-Sur. (Trabajo de Grado). Universidad Autónoma del Perú, Lima. http://repositorio.autonoma.edu. pe/bitstream/AUTONOMA/124/1/ROSALES\%20FERNANDEZ.pdf

Sarubbi De Rearte, E. y Castaldo, R. I. (2013). Factores causales del estrés en los estudiantes universitarios. $V$ Congreso Internacional de Investigación y Práctica Profesional en Psicología. XX Jornadas de Investigación Noveno Encuentro de Investigadores en Psicología del MERCOSUR. Facultad de Psicología - Universidad de Buenos Aires, Buenos Aires. https://www.aacademica.org/000-054/466.pdf

Sicre, E. y Casaro, L. (2014). Estrategias de afrontamiento en estudiantes de Psicología. Revista de Psicología, 20(10), 61-72. http://revistas.pucp.edu.pe/ index.php/psicologia/article/view/6849 
Somerfield, M. R. \& McCrae, R. R. (2000). Stress and coping research: Methodological challenges, theoretical advances, and clinical applications. American Psychologist, 55(6), 620-625. https://www.ncbi.nlm.nih.gov/pubmed/10892204

Suárez-Montes, N. y Díaz-Subieta, L. B. (2015). Estrés académico, deserción y estrategias de retención de estudiantes en la educación superior. Revista de Salud Pública, 17(2), 300-313. http://www.scielo.org.co/pdf/rsap/v17n2/v17n2a13.pdf

Ticona, S., Paucar, G. y Llerena, G. (2010). Nivel de estrés y estrategias de afrontamiento en estudiantes de la facultad de enfermería - UNSA Arequipa 2006. Enfermería Global, 9(2), 1-18. https://www.redalyc.org/articulo.oa?id=365834755007

Toribio-Ferrera, C. y Franco-Bárcenas, S. (2016). Estrés académico: el enemigo silencioso del estudiante. Salud y Administración, 3(7), 11-18. http://www.unsis. edu.mx/revista/doc/vol3num7/A2_Estres_acad.pdf

Villarroel, Z, A. y González, R. M. (2015). Intervención cognitivo conductual y centrada en soluciones para disminuir el estrés académico en estudiantes universitarios. Revista Electrónica de Psicología Iztacala, 18(4), 1363-1387. http://www.revistas. unam.mx/index.php/repi/article/view/53434

Vogel, S. \& Schwabe, L. (2016). Learning and memory under stress: implications for the classroom. Science of Learning, 1, 1-10. https://www.nature.com/articles/ npjscilearn 201611

Zárate, N. E., Soto, M. G., Castro, M. L. y Quintero, J. R. (2017). Estrés académico en estudiantes universitarios: medidas preventivas. Revista de la Alta Tecnología y la Sociedad, 9(4), 92-98. http://sistemanodalsinaloa.gob.mx/ archivoscomprobatorios/_11_articulosrevistasindexadas/22159.pdf 